\title{
Model Design of Indoor Visible Light MIMO Communication System Based on Space Shift Keying
}

\author{
Xiangwen $\mathrm{Li}^{1}$, Liping $\mathrm{Li}^{2}$, Ling Zhou ${ }^{1}$ Shuang Zhang and Yi-he Liu ${ }^{3, *}$ \\ ${ }^{I}$ The Engineering \& Technical College of Chengdu University of Technology, Leshan, 614000, P.R. China $;{ }^{2}$ China Ship \\ Research Institute of Beijing Huan Jia Communication Technologies Company, Beijing, 100085, P.R. China; ${ }^{3}$ College \\ of Computer Science, Neijiang Normal University, Neijiang,641000, P.R. China
}

\begin{abstract}
In order to enhance the reliability of the indoor visible light MIMO wireless communication system and improve the ability of anti-interference between channels of the system, this paper introduces the new model of Space Shift Keying in the radio frequency communication into the visible light MIMO communication. The simulation analysis shows, the MIMO system of SSK has almost similar performance of repetitive coding which effectively overcomes the problems in the conventional system such as interference between channels and complexity of decoding. Therefore, it is a suitable implementation plan for low-complexity indoor visible light MIMO wireless communication system
\end{abstract}

Keywords: Multiple-in-multiple-out, space shift keying, visible light communication, wireless communication.

\section{INTRODUCTION}

Recently, with the increasing demand for the mobility, security, confidentiality and personalization of mobile communication, people have higher requirements for communication quality, cost, speed and capacity. The conventional radio frequency radio communication technology gradually fails to meet the new requirements, and a new communication design scheme is urgently needed to solve the problems in indoor wireless optical communication. Through the use of white LED, the indoor visible light wireless communication can meet the demand of not only communication, but also the Indoor lighting. The luminous intensity of LED light can vary considerably along with the variation of electric signal. In visible light wireless communication, the spectral bandwidth of the optical carrier is over 10000 times larger than that of the radio frequency communication. Therefore, in the future market, it has huge potentials and broad market prospect.

In order to improve the spectrum efficiency and the link quality of wireless communication system, it is effective to adopt several communication antennas to send and receive data at the receiving terminal and the sending terminal of the system respectively-using multiple input multiple output communication system (MIMO). Using multiple communication antennas to send signals at the sending terminal or to receive signals at the receiving terminal helps to improve the system performance and capacity. However, it still has such problems as interference between channels and complex decoding process.

In contrast to the indoor visible light point-to-point wireless communication technique, the MIMO wireless communication installs several white LED lights and several photoelectric detectors in the room, which can not only realize wider indoor signal coverage, but also better indoor illumination, thus achieving the integration of indoor illumination and communication. The OOK modulation technique is used in indoor visible communication which has simple hardware implementation and integration. Therefore, with better effect in compromising complexity and performance of communication systems, it is widely applied in the indoor visible communication.

\section{MODEL DESIGN WITH SPATIAL MODULATION TECHNIQUE}

The MIMO wireless communication system is configured with multiple sending antennas and/or multiple receiving antennas. Assuming the number of the sending antennas and receiving antennas is $\mathrm{N}_{\mathrm{t}}$ and $\mathrm{Nr}$ in the system, the model of MIMO wireless communication system is shown in Fig. (1).

The theory of MIMO communication is to combine the data signals in the sending antennas and receiving antennas. The use of spatial modulation(SM) in MIMO communication can enhance the ability of the anti-interference between channels in MMO wireless communication system and reduce complexity in hardware implementation at the sending terminal and the receiving terminal.

The spatial modulation technical plan in MIMO communication is similar to the repetitive coding technical plan for they both adopt multiple transmitters to send data at the sending terminal of the communication system. Their difference lies in the idea that, compared to other modulation, the spatial modulation not only transmits and modulates the data onto the carrier, but also makes some data mapped into space. 


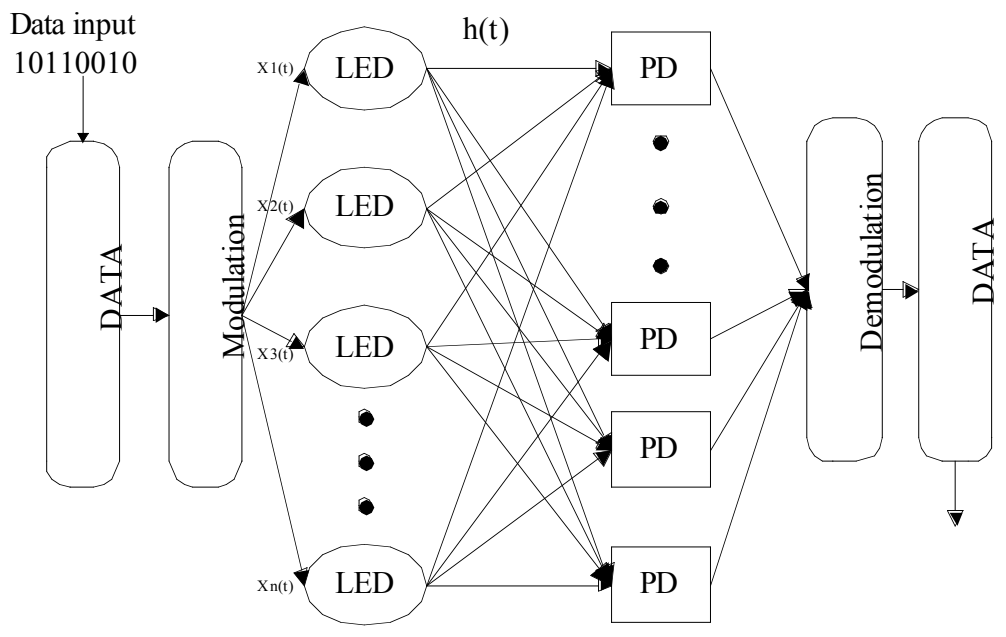

Fig. (1). Model of indoor visible light MIMO wireless communication.

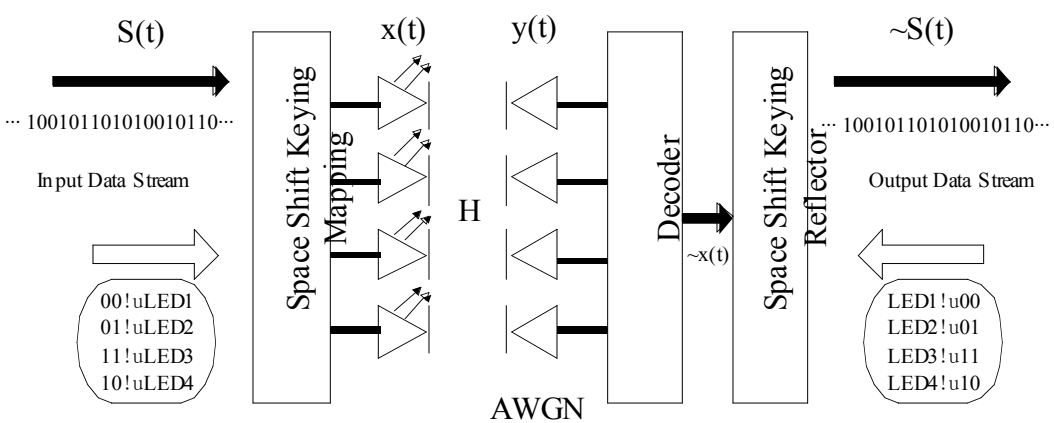

Fig. (2). Model of indoor visible light MIMO wireless communication based on SSK.

In the model of indoor visible light MIMO wireless communication, simple technique of intensity modulation and direct detection is adopted. That is, there are $\mathrm{N}_{t}$ white LED light sources at the sending terminal and $\mathrm{Nr}$ photoelectric detectors at the receiving terminal. The model of indoor visible light MIMO wireless communication system based on SSK is shown in Fig. (2).

As is shown in Fig. (2), the number of the sending antennas and receiving antennas are supposed to be 4 for simplicity to make a $4 \times 4$ SSK-MIMO communication system. $\mathrm{N}_{\mathrm{t}}$ can be other values, but in order to distinguish the points of antenna array properly in space domain constellation chart, the number of sending antennas in the sytem $\mathrm{N}_{\mathrm{t}}$ should be function of integer power of 2 , because the data transmitted in the visible light wireless communication are binary bit data. The bit data stream which has been sent can be grouped at any moment based on the sending antenna units described above, t. In Fig. (2), $\mathrm{N}_{\mathrm{t}}$, the sending antennas, is 4, so the bit data stream are grouped according to the equation $\log _{2} \mathrm{~N}_{\mathrm{t}}=2$. Through the modulating of SSK, the sending bit data stream enters into the space keying mapping. Every two bits $\left(\log _{2} \mathrm{Nt}\right)$ are mapped onto the sending antenna of corresponding index number. At the exact moment, the activated sending antenna transmit optical signal, whose power intensity can achieve $\mathrm{P}_{\mathrm{t}}$, while other antennas don't transmit optical signals. Through the light MIMO Wireless channel $\mathrm{H}$, the optical signal is transmitted to the receiving terminal, which consists of multiple photoelectric detectors. PD detects the received optical power. Then, original data information flow is regained through decoding and SSK demodulation. The receiving signal $\mathrm{y}(\mathrm{t})$ is shown by Equation (1).

$$
y(t)=\sqrt{\rho} H(t) * x(t)+n(t)
$$

In Equation (1), * stand for Convolution and $\rho$ is average SNR in every receiving antenna. $\mathrm{H}(\mathrm{t})$ is $\mathrm{N}_{\mathrm{r}} \times \mathrm{N}_{\mathrm{t}}$ wireless channel matrix of light MIMO, and its matrix elements is $\mathrm{h}_{\mathrm{ij}}(\mathrm{t})$, which means the impulse response of optical wireless link channel, between the sending antenna (i) and receiving antenna (j). The path loss is $h_{j i}=\int_{-\infty}^{+\infty} h_{j i} t d t$. Assuming the transmitting is LOS model, the $\mathrm{h}_{\mathrm{ij}}(\mathrm{t})$ can be calculated by Equation (2).

$h_{j i}=\left\{\begin{array}{c}\frac{(m+1) A}{2 \pi_{j i}^{2}} \cos ^{m}\left(\Phi_{j i}\right) T_{s}\left(\Psi_{j i}\right) g\left(\Psi_{j i}\right) \cos \left(\Psi_{j i}\right), 0 \leq\left(\Psi_{j i}\right) \leq \Psi_{c} \\ 0\end{array}\right.$

\section{DECODING ALGORITHM OF VISIBLE LIGHT MIMO COMMUNICATION SYSTEM IN THE SPACE SHIFT KEYING ROOM}

With the Space Shift Keying, the indoor visible light MIMO wireless communication avoids the conventional modulation model, and has the advantages of simplifying the hardware implementation in the sending and receiving ter- 

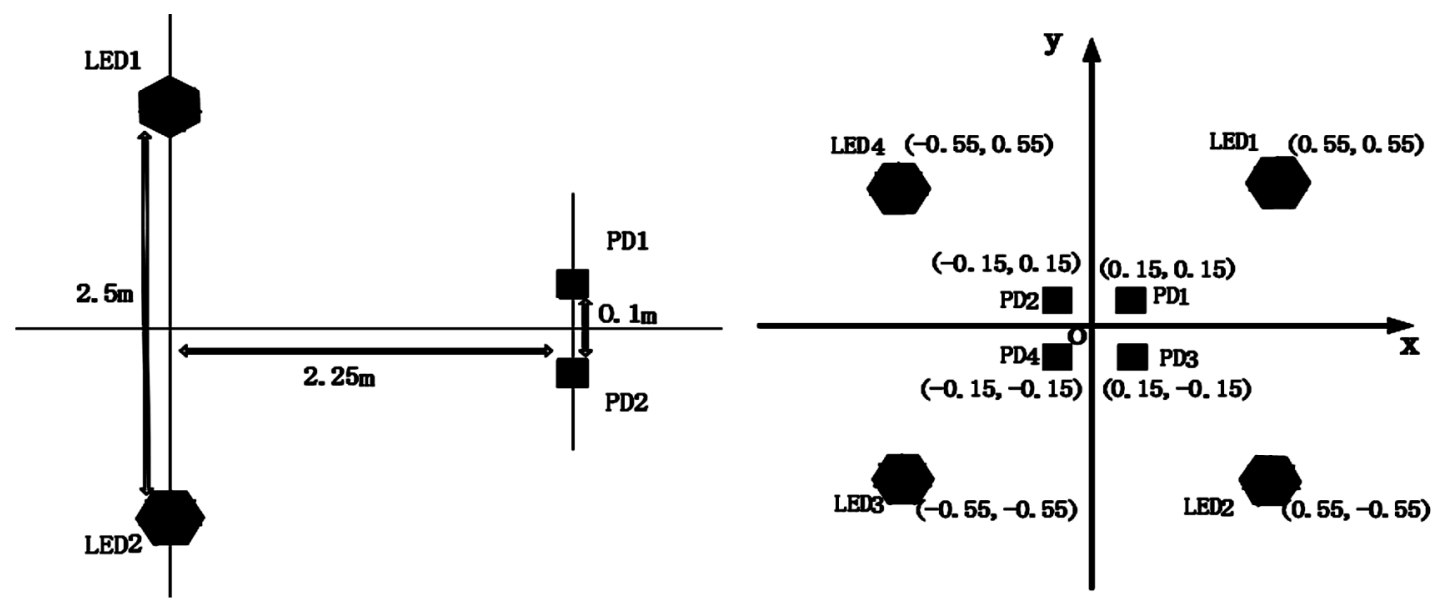

Fig. (3). Spatial geometrical layout of sending and receiving terminals in the light MIMO wireless communication system: (a) $2 \times 2-\mathrm{MIMO}$ communication system (b) $4 \times 4-$ MIMO communication system.

minals, reducing the complexity of decoding algorithm, and providing better data transmission rate and communication quality. The photoelectric detectors at the receiving terminal designed in this paper convert the received optical signals into electrical signals, and transmit them to the optimal receiver to decode and send the index sequence number of the sending antenna array. In order to regain the original signal, the receiver is assumed to have ideal time synchronization and know the complete channel state information(CSI). The channel impulse response can be obtained through the channel estimation. By the use of intensity modulation and direct detection, the optical power signal is non-negative value. The index sequence number of the sending antennas which the optimal estimated by the receiver is shown in Equation (3).

$i=\arg \min \sqrt{\rho}\left\|h_{i} x_{i}\right\|_{F}^{2}-2\left(y^{T} h_{i} x_{i}\right)$

In the equation, $i$ is the index sequence number of white LED sources, estimated by optimal receiver. $\mathrm{H}$ is $\mathrm{N}_{\mathrm{r}} \times \mathrm{N}_{\mathrm{t}}$ MIMO wireless channel matrix. $x$ is the column vector signal, transmitted from sending signal matrix $x(t)$ at each instantaneous point. In the vector signal $\mathrm{x}, \mathrm{P}_{\mathrm{t}}$ means light power, standing for the corresponding value of the activated transmitted antenna, while the non-activated antenna is $0, p_{\mathrm{y}}$ $(\mathrm{y} \mid \mathrm{x}, \mathrm{H})$ stands for conditional probability density function for $\mathrm{y}$ signal, under the condition of transmitting signal is $\mathrm{x}$ and transmission channel matrix is $\mathrm{H}$, which can be shown in equation 4 .

$p_{y}(y \mid x, H)=\pi^{-N_{r}} \exp \left(-\|y-\sqrt{\rho} H x\|_{F}^{2}\right)$

Through the well-known uniform boundary technology, the upper bound error rate of the SSK system can be deduced. The bit error rate can be shown in equation 5 .

$B E R=\sum_{k=1}^{N_{t}} \sum_{v \neq k=1}^{N_{t}} \frac{N(k, v)}{N_{v}} P E P(k \rightarrow v)$

In Equation 5, $\mathrm{N}(\mathrm{k}, \mathrm{v})$ is the error bit number, calculated by the difference between the sending antenna index number $\mathrm{V}$ restored from the received signal and the actual antenna index number $\mathrm{k}$. PEP $(\mathrm{k} \rightarrow \mathrm{v})$ stands for pair wise error probability, resulted from the error judgment of sending antenna $\mathrm{K}$ as $\mathrm{V}$, which is shown in Equation (6).
$\operatorname{PEP}\left(k \rightarrow v \mid h_{k}, h_{v}\right)=Q\left(\sqrt{\frac{\pi}{4}}\left\|h_{k}-h_{v}\right\|_{F}^{2}\right)$

In the equation, $Q(x)=\left(\frac{1}{2 \pi}\right) \int_{x}^{+\infty} \exp \left(-\frac{t^{2}}{2}\right) d t$ is function $\mathrm{Q}$.

In the simulation models, the $2 \times 2$-MIMO and $4 \times 4$ MIMO communication system are chosen. The modulation bandwidth of white light LED source is set as $6 \mathrm{MHz}$ In the $2 \times 2$-MIMO system, the distance between the two LED sources in the sending terminal is $1.6 \mathrm{~m}$, and the two photoelectric detectors in the receiving terminal is $10 \mathrm{~cm}$. The layout of the sending and receiving terminal of the system is shown in Fig. (3a). The radiant half power angle of LED source $\left(\Phi_{1 / 2}\right)$ is $45^{\circ}$, and the photoelectric conversion rate of $\mathrm{PD}(\mathrm{r})$ is $0.5 \mathrm{~A} / \mathrm{W}$. The detection area of PD (A) is $1 \mathrm{~cm}^{2}$, and the angle of detecting field of view $(\Psi)$ is $90^{\circ}$. In the $4 \times 4$ MIMO system, the distance between the sending terminal and the receiving terminal coordinate plane is $2.25 \mathrm{~m}$. The radiant half power angle of LED source $\left(\Phi_{1 / 2}\right)$ is 15 . The photoelectric conversion rate and detection area are equal to those in $2 \times 2$-MIMO system. In $4 \times 4-$ MIMO system, the top view of space geometry layout of the LED sources and PD is shown in Fig. (3b). The mapping relationship between the data bit stream in space mapping device and the index number of LED sources is shown in Fig. (2).

According to Equation (5), the channel matrix gain $(\mathrm{H})$ in $2 \times 2$-MIMO in Fig. (3b) can be gained as in Equation (7):

$H=1.2 e-8 *\left[\begin{array}{ll}1.2505 & 1.1450 \\ 1.1450 & 1.2505\end{array}\right]$

While the gain $(\mathrm{H})$ in $4 \times 4-\mathrm{MIMO}$ is gained in Equation (8) as

$H=1.2 e-6 *\left[\begin{array}{llll}0.5525 & 0.0566 & 0.1255 & 0.0566 \\ 0.0566 & 0.1255 & 0.0566 & 0.5525 \\ 0.0566 & 0.5525 & 0.0566 & 0.1255 \\ 0.1255 & 0.0566 & 0.5525 & 0.0566\end{array}\right]$

According to Equation 7 and Equation 8, the simulation graphics of error rate performance in $2 \times 2$-MIMO and $4 \times 4$ MIMO can be obtained. The process is shown in Fig. (4). 


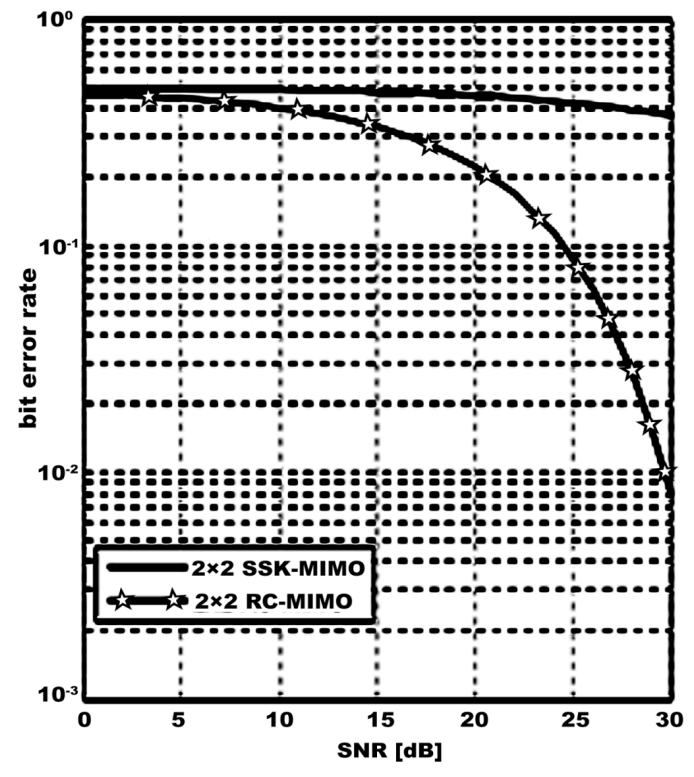

(a)

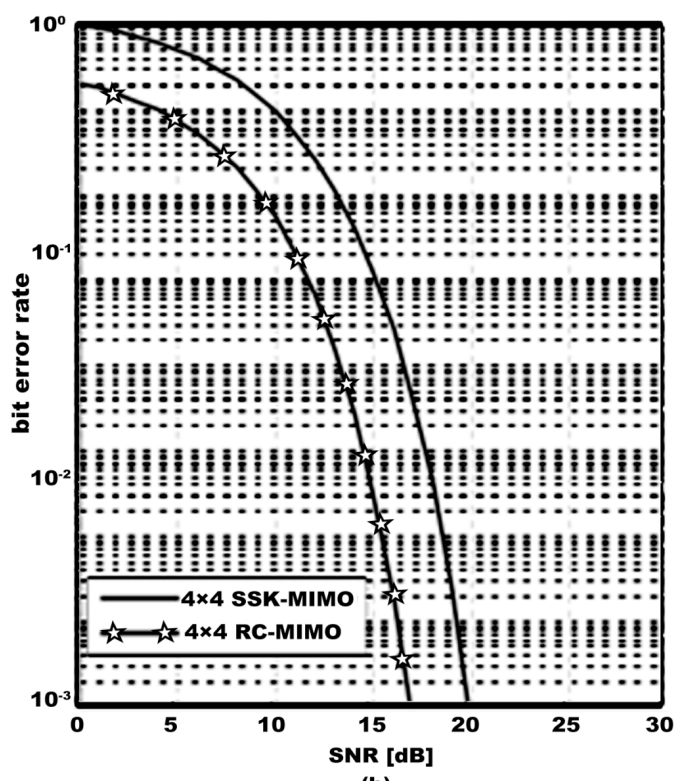

(b)

Fig. (4). Comparison of bit error rate between SSK and RC MIMO System (a) 2×2-MIMO system (b) 4×4-MIMO system.

From the result, it can be concluded that in the $2 \times 2$ MIMO system, the system performance of the repetitive coding scheme is superior to SSK. However, if the numbers of the antenna in the sending and receiving terminals are added in the $4 \times 4$-MIMO system, the performance of MIMO system with SSK is close to that of the repetitive coding plan. With the same error rate, repetitive coding scheme has the edge of $3 \mathrm{~dB}$, but the data rate of SSK is twice more than that of the repetitive coding scheme under OOK modulation. From the above analysis of the advantages of the SSK MIMO system, it can be concluded that in the indoor visible light MIMO wireless communication system, the technique of SSK MIMO can be used as the implementation scheme of the low- complexity MIMO wireless communication system.

\section{CONCLUSION}

Based on the model of indoor visible light MIMO wireless communication system with Space Shift Keying, this paper makes an analysis of the decoding algorithm in the system, provide the bit error rate of the system and compares its performance with that of the system with repetitive coding scheme. The simulation analysis shows that the performance of SSK MIMO system is similar to that of the repetitive coding plan. It can be applied in the low-complexity indoor visible light MIMO wireless communication.

\section{CONFLICT OF INTEREST}

The authors confirm that this article content has no conflict of interest.

\section{ACKNOWLEDGEMENTS}

This work presented in this paper is supported by The Key Fund Project of Sichuan Provincial Department of Education under Grant13ZA0003, Grant 14ZB0360, Grant 14ZB0363, Grant 14ZB0352; The Sichuan Province Department of Science and Technology under Grant 2015JY0119 ;the Science and Technology Development Fund of Macau (FDCT) under Grant 014/2007/A1, Grant 063/2009/A, and Grant 024/2009/A1; the Research Committee of the University of Macau, under Grant RG072/0910S/MPU/FST; the Key Fund Project of Leshan Science and Technology Bureau (14GZD046).

\section{REFERENCES}

[1] Takase D, Ohtsuki T. "Optical wireless MIMO communications (OMIMO)”, IEEE Global Telecommunications Conference, vol.2 , 2004, pp. 928-932.

[2] Jeganathan J, Ghrayeb A, Szczecinski L, et al. Space shift keying modulation for MIMO channels [J]. IEEE Transactions on Wireless Communications, vol. 8(7), pp. 3692-3703, 2009,

[3] Komine T, Nakagawa M. Fundamental analysis for visible-light communication system using LED lights [J].IEEE Transactions on Consumer Electronics, vol. 50(1), pp. 100-107, 2004.

[4] Jeganathan J, Ghrayeb A, Szczecinski L, et al. Space shift keying modulation for MIMO channels [J]. IEEE Transactions on Wireless Communications, vol. 8(7), pp. 3692-3703. 2009.

[5] Mesleh R, Mehmood R, Elgala H, et al. "Indoor MIMO optical wireless communication using spatial modulation ," IEEE International Conference on Communications (ICC 2010), 2010, pp. 1-5. 